\title{
Emerging Agrigenomics Approaches in Stress Plant Biology
}

\author{
Vinay Sharma \\ Department of Bioscience \& Biotechnology \\ Banasthali University, P.O. Banasthali Vidyapith- 304022 (Rajasthan), INDIA
}

Biotic stress owing to infection of the plants by phytopathogens causes immense losses in crop yield, up to $12 \%$ annually. Abiotic stress is another major cause of crop losses worldwide, reduces average yield by more than $50 \%$ for major crops. Globally, $22 \%$ of the agricultural land is saline and drought stress contribute to $26 \%$ in crop losses; in fact only $10 \%$ area is not affected by any stress factors. In recent times, high-throughput technologies (viz. next gene sequencing) have produced huge amount of genomic information and consequently the genome sequences of a large number of micro to higher organisms have been completed. The whole genome sequences of a large number of crop plants and their bacterial and fungal pathogens are now available. With the ever growing sequence data, the functional prediction/ annotation of proteins which is of paramount importance, has become a challenging task. Although the wet laboratory experiments identify the function of proteins correctly, these are time-consuming and costly and impractical to deal with the enormous genome data. Consequently several computational approaches based on protein-protein interaction (PPI) and others have been developed. With the availability of the host-pathogen sequences, the tools of bioinformatics in developing drugs against specific plant pathogens has great potential and have been currently being explored by us in the prediction of drug targets against Pseudomonas syringae pv. phaseolicola which is a major plant pathogen and infects a wide range of plants, including the model plant Arabidopsis thaliana and other major crops causing devastating diseases responsible for huge economic losses including halo blight disease in beans. We predicted drug targets in this important bacterial pathogen by exploiting novel bioinformatics approaches (OMICS, 16:700-706). We used gene sequence information for prediction of drug targets in P. syringae pv. phaseolicola using subtractive genomics to exclude common proteins with the host plant Phaseolous vulgaris, and then the codon usage biasing for the detection of high expression genes which was validated on the basis of cDNA microarray data. We also used a second subtraction step to avoid accidental targeting of 'friendly species', the nitrogen fixing bacteria Rhizobium etli. In order to understand abiotic stress, we used a combination of wet lab and in silico approaches in Sorghum bicolor, the fifth most important cereal crop in the world for food, feed and biofuels and further the small size genome $(\sim 730 \mathrm{Mb})$ of sorghum makes it an attractive model plant for functional genomics of $\mathrm{C} 4$ grasses. We used shortest path (SP) analysis method on Gene Ontology (GO) hierarchy for functional identification of putative uncharacterized abiotic stress responsive proteins isolated by us using gel electrophoresis and MALDI-TOF and suggested a distinct mechanism of stress adaptation in sorghum plant (Physiol. Molecular Biol. of Plants, 18: 209216). This novel approach has the potential to be applied in identifying proteins involved in abiotic and biotic stress in other crops. This paper has reviewed various evolving agrigenomics approaches in identifying genes/ proteins in stress plant biology. 